\title{
YouTube as a source of information on COVID-19 and rheumatic disease link
}

\author{
Burhan Fatih Kocyigit ${ }^{1}$ (D) $\cdot$ Mazlum Serdar Akaltun $^{2}$ (D) $\cdot$ Ahmet Riza Sahin $^{3}$ (D) \\ Received: 28 April 2020 / Revised: 28 April 2020 / Accepted: 15 May 2020 / Published online: 23 May 2020 \\ (C) International League of Associations for Rheumatology (ILAR) 2020
}

\begin{abstract}
Introduction/objectives The current 2019 novel coronavirus outbreak is continuing to spread rapidly despite all efforts. Patients with rheumatic disease may have higher levels of anxiety due to their disease characteristics and medications. The web-based platforms are widely used sources for gaining medical information. YouTube presents a wide range of medical information, but there are concerns on its quality. Therefore, we aimed to evaluate the quality of the YouTube videos about COVID-19 and rheumatic diseases link.

Method This is a descriptive study. A total of 360 videos listed by the YouTube search engine (www.youtube.com) in response to six search terms were evaluated. The Global Quality Scale (GQS) was performed to evaluate video quality. Three groups were formed according to GQS scores: high quality, moderate quality, and low quality. Video parameters were compared between these groups.

Results After the exclusion criteria, 46 videos were reviewed. Of the videos, $41.4 \%(n=19)$ were of high-quality group, $21.7 \%$ $(n=10)$ were moderate-quality group, and $36.9 \%(n=17)$ were of low-quality group. Significant difference was detected between the quality groups in terms of views per day $(p=0.004)$. No significant difference was detected in comments per day $(p=0.139)$ and like ratio $(p=0.232)$.

Conclusions Besides high-quality videos, there were substantially low-quality videos that could cause misleading information to spread rapidly during the pandemic. Videos from trustworthy sources such as universities, academics, and physicians should be kept in the foreground.

\section{Key Points}

-Web-based platforms have become an important source of health-related information. One of the most important online sources is YouTube because it is easy accessible and free.

- Of the videos evaluating the link between COVID-19 and rheumatic diseases, $41.4 \%(n=19)$ were of high quality.

-The main sources of high-quality videos were academics/universities and physicians.

-The most frequently discussed topics in videos were the place of hydroxychloroquine in the treatment of COVID-19 and whether to continue the use of existing rheumatological drugs.
\end{abstract}

Keywords COVID-19 $\cdot$ Pandemic $\cdot$ Rheumatic diseases $\cdot$ SARS-CoV-2 $\cdot$ YouTube

Burhan Fatih Kocyigit bfk2701@hotmail.com

Mazlum Serdar Akaltun mazlum_akaltun@hotmail.com

Ahmet Riza Sahin drahmetrizasahin@gmail.com
1 Faculty of Medicine, Department of Physical Medicine and Rehabilitation, Kahramanmaraș Sütçü İmam University, Kahramanmaraş, Turkey

2 Faculty of Medicine, Department of Physical Medicine and Rehabilitation, Gaziantep University, Gaziantep, Turkey

3 Faculty of Medicine, Department of Infectious Diseases and Clinical Microbiology, Kahramanmaraș Sütçü İmam University, Kahramanmaraş, Turkey 


\section{Introduction}

A new infectious disease detected in Wuhan, Hubei province, China, at the end of 2019, spread throughout the whole world and rapidly caused a "pandemic." The World Health Organization reported SARS-CoV-2, a virus of the Coronaviridae family, to be the infection agent $[1,2]$. This agent leads to severe pneumonia, called COVID-19, in humans.

During this period, anxiety and maladaptive behaviors increased in patients undergoing chronic immunosuppressive therapy with the thought that there was a lack of immune response against viruses [3]. Patients with rheumatic diseases may feel greater concern as the characteristics of the disease can make them more vulnerable to infectious diseases. In addition, the fact that patients are actively using non-steroidal anti-inflammatories, corticosteroids, and synthetic and biological disease-modifying agents may increase these concerns [4]. Therefore, it is inevitable that patients will increasingly seek alternative sources of health-related information.

In the digitalized world, web-based platforms are probably the most easily accessible health-related information sources for the community. YouTube has grown into one of the most popular social platforms in the world with more than one billion Internet consumers and 6 billion hours of viewing time every month [5]. YouTube includes a large number of videos on the pathogenesis, prevention, diagnosis, and treatment options of several health problems [6-9]. YouTube videos related to Ebola were viewed millions of times during the 2014 outbreak [10]. During disease outbreaks, online platforms should be considered a "double edged sword," which can lead to positive and negative consequences. While the dissemination of accurate YouTube-based information may contribute to controlling the outbreak, misleading information may cause panic in society and increase the spread of disease. YouTube's main handicap in this regard is that it does not include a regulatory mechanism to control the content quality of the uploaded videos.

To the best of our knowledge, there have been no previous studies assessing YouTube videos providing information on COVID-19 related to rheumatic diseases. Therefore, the aim of this study was to evaluate the quality of YouTube videos about COVID-19 in relation to rheumatic diseases. It was also aimed to identify video sources providing high-quality information and to determine which topics were covered more in these videos.

\section{Materials and methods}

This was planned as a descriptive study, and the possible search terms were discussed in the first stage. A total of six search terms were determined as "COVID-19 rheumatology,
SARS CoV-2 rheumatology, coronavirus 2019 rheumatology, COVID-19 arthritis, SARS CoV-2 arthritis, and coronavirus 2019 arthritis." These search terms were used on YouTube (www.youtube.com) on 2 April 2020 to determine videos that could be evaluated in the study. The browser search history was deleted before the study to minimize the effect of past Internet use on search results. Video listings are made based on view counts which enables the most viewed videos to be listed on the first page. Although there are studies assessing all videos, the most common method is to work on a fixed sample size [11]. It has been reported that over $90 \%$ of Internet users review videos on the first 3 pages of query results [12]. Therefore, the videos on the first three pages (60 videos) for each search term were reviewed. The 360 videos were recorded in a file for future analysis because search results on YouTube are constantly changing. This sample selection method has been used in similar studies in literature [6, 13, 14]. Videos in a language other than English, duplicate videos, off topic videos, and videos without audio were excluded from the study.

\section{Assessment of quality}

The quality of the videos was assessed using the Global Quality Scale (GQS) which is a 5-point scale of 1-5 points and has been used for quality assessments in similar studies $[6$, $9,13,15]$. Video flow, usefulness, and quality can be evaluated using the GQS, with scores applied of 4-5 points indicating high quality, 3 points indicating moderate quality, and 1-2 points indicating low quality. The following scoring system was used in this study:

- 1 point: The video is of poor quality, poor flow, lacking most information, and therefore not useful for patients.

- 2 points: The video is generally of poor quality, and although some information is given, it is of limited use for patients.

- 3 points: The video is of moderate quality, and some important information is sufficiently discussed. In these videos, accurate and incorrect information are presented in a balanced manner. However, high-quality information is provided together with misleading information.

- 4 points: The video is of good quality and good flow. The video is useful for patients, covering the most relevant information and presenting accurate information to a large extent, but it may include minor deficiencies.

- 5 points: The video is of excellent quality and excellent flow and is very useful for patients. These videos include completely accurate information [16].

All the videos were independently evaluated by two physicians (BFK, MSA). The Kappa coefficient was used for the determination of inter-rater consistency. Videos with 
inconsistency in terms of GQS scores (between BFK and MSA) were identified. These were then evaluated by another physician (ARS), and consensus was reached.

\section{Assessment of reliability}

The modified DISCERN tool (DS) was applied to assess reliability. The modified DS was adapted from the original version and includes five yes-no questions [15]:

- Is the video clear, concise, and understandable?

- Are reliable sources of information used?

- Is the information presented balanced and unbiased?

- Are additional sources of information listed for patient reference?

- Are areas of uncertainty/controversy mentioned?

Each "yes" answer is scored as 1 point and each "no" answer as 0 point. Thus, the score ranges from 0 to 5 points with higher scores indicating greater reliability.

\section{Video parameters}

The upload date, video duration, view counts, likes, dislikes, and comments were recorded for all videos. The values for views per day were calculated by dividing the total number of views by the total number of days on YouTube. The like ratio was calculated as like / [like + dislike].

\section{Video sources}

Video sources were categorized under eight headings: (1) society/non-profit organization, (2) physician, (3) healthrelated website, (4) university/academic, (5) patient/ independent user, (6) non-physician healthcare personnel, (7) pharmaceutical company, and (8) news agency.

\section{Coding of video content}

It was recorded whether the video addressed the following topics: symptoms of COVID-19, relationship between rheumatological diseases and risk of COVID-19, whether to continue the use of existing rheumatological drugs, COVID-19 prevention practices for those with rheumatological disease, the place of hydroxychloroquine in the treatment of COVID19 , conditions that should be consulted to a doctor or hospital, and psychological effects of COVID-19 on rheumatology patients.

\section{Ethics statement}

Videos which are publicly available on YouTube were assessed, and no human participants/animals were included. Therefore, Ethics Committee approval was not required for this study. Similar studies have also followed the same path $[6,17,18]$.

\section{Statistical analysis}

Data obtained in the study were analyzed statistically using the Statistical Package for the Social Sciences version 20.0 software (SPSS Inc., Chicago, IL, USA). Descriptive data were stated as number, percentage, and median (minimummaximum) values. Conformity of the data to normal distribution was checked using the Shapiro-Wilk test. The agreement between the two physicians was assessed using the Kappa coefficient. Comparisons between low-, moderate-, and high-quality groups were performed using the KruskalWallis test. A value of $p<0.05$ was accepted as statistically significant.

\section{Results}

Of the total 360 videos, 176 off topic videos, 113 repetitive videos, 22 videos in a language other than English, and 3 videos with sound quality problems were excluded from the study. After application of the inclusion and exclusion criteria, a total of 46 videos were identified for evaluation in the study. The median duration of the videos was $403.5 \mathrm{~min}$ (range, 31$3699 \mathrm{~min}$ ). The median view count and number of comments were 589.5 (range, 21-635,561) and 2.5 (range, 0-1972), respectively. The general features of the videos are presented in Table 1.

Of the videos, $41.4 \%(n=19)$ were of high quality, $21.7 \%$ $(n=10)$ were of moderate quality, and $36.9 \%(n=17)$ were of low quality according to the GQS. The Kappa score for interrater agreement was 0.74 . Of the videos, $100 \%(n=2)$ produced by academics/universities and $56.3 \%(n=9)$ produced

Table 1 General features of the videos

\begin{tabular}{lc}
\hline Video features & Median (min-max) \\
\hline Duration (seconds) & $403.5(31-3699)$ \\
View count & $589.5(21-635,561)$ \\
Number of comments & $2.5(0-1972)$ \\
Total likes & $14(0-8800)$ \\
Total dislikes & $0(0-308)$ \\
\hline
\end{tabular}

min Minimum, $\max$ maximum 
Table 2 Categorization of the videos according to sources, $n$ (\%)

\begin{tabular}{lllll}
\hline Source & Low quality & Moderate quality & High quality & Total \\
\hline Society/non-profit organization & $3(50)$ & $0(0)$ & $3(50)$ & 6 \\
Physician & $1(6.2)$ & $6(37.5)$ & $9(56.3)$ & 16 \\
Health-related website & $2(20)$ & $4(40)$ & $4(40)$ & 10 \\
Academic/university & $0(0)$ & $0(0)$ & $2(100)$ & 2 \\
Patient/independent user & $4(100)$ & $0(0)$ & $0(0)$ & 4 \\
News agency & $7(87.5)$ & $0(0)$ & $1(12.5)$ & 8 \\
\hline
\end{tabular}

$n$ number, $\%$ percentage by physicians were of high quality. Low-quality videos were determined as $100 \%(n=4)$ of those produced by patients/ independent users and $87.5 \%(n=7)$ of the videos produced by news agencies. The distribution of the video quality groups according to the uploading sources is presented in Table 2.

Significant differences were detected between the quality groups in terms of DS scores and views per day $(p<0.0001$, $p=0.004)$. The highest scores were found in the high-quality group. No significant differences were determined in respect of comments per day $(p=0.139)$ and like ratios $(p=0.232)$ according to video quality (Table 3 ).

When the content of the videos was examined, $45.6 \%(n=$ 21 ) provided information on "the place of hydroxychloroquine in the treatment of COVID-19," and $41.3 \%(n=19)$ provided information on "whether to continue the use of existing rheumatological drugs." The distribution of the videos according to content is presented in Table 4.

\section{Discussion}

YouTube is a powerful and free platform which can reach a large part of society during a pandemic. As a result of rapid adaptation to digital life, the use of Internet platforms such as YouTube can be expected to increase as a source of medical information. However, the structure of YouTube allows it to present videos providing high-quality/useful information and misleading/inaccurate information at the same time. The spread of misleading/inaccurate information during a pandemic can increase anxiety and panic, leading to undesirable results. Therefore, it was considered that it would be useful to evaluate Internet-based information during this COVID-19 pandemic. The study results demonstrate that less than half of the YouTube videos focusing on the relationship between COVID-19 and rheumatological diseases were of high quality. The main sources of the high-quality videos were academics/universities and physicians, whereas the videos presented by patients/independent users and news agencies were of predominantly low quality.

Of the total videos, $41.4 \%(n=19)$ were of high quality, $21.7 \%(n=10)$ were of moderate quality, and $36.9 \%(n=17)$ were of low quality. Khatri et al. [19] evaluated YouTube videos on the novel coronavirus outbreak and reported that $67 \%$ of the videos in English were useful. During the Zika virus pandemic and the $\mathrm{H} 1 \mathrm{~N} 1$ influenza pandemic, $70.3 \%$ and $61.3 \%$ of the videos, respectively, were reported to be useful and informative [20, 21]. However, there are also studies reporting lower rates of high-quality/useful videos outside of the pandemic $[13,22]$. In the current study, several factors may have played a role in detecting relatively lower rates of high-quality/useful videos compared with other pandemic studies. In other studies, the pandemic was evaluated as a whole, whereas in this study, there was particular focus on the relationship between the pandemic and rheumatic diseases. This may have affected the results. The current study sample was relatively small compared with other pandemic studies, and the assessment of videos is subjective, and different criteria may have been used in other evaluations.
Table 3 DS, views per day, comments per day, and like ratio of videos according to video quality

\begin{tabular}{llllr}
\hline Video quality & $\begin{array}{l}\mathrm{DS}^{\mathrm{a}} \\
\text { Median (min-max) }\end{array}$ & $\begin{array}{l}\text { Views per day }^{\mathrm{b}} \\
\text { Median (min-max) }\end{array}$ & $\begin{array}{l}\text { Comments per day }^{\mathrm{c}} \\
\text { Median (min-max) }\end{array}$ & $\begin{array}{l}\text { Like ratio }^{\mathrm{d}} \\
\text { Median (min-max) }\end{array}$ \\
\hline Low & $2(1-3)$ & $35.80(2-241.40)$ & $0.2(0-1.6)$ & $0.94(0-1)$ \\
Moderate & $2(1-4)$ & $41.93(8-166.63)$ & $0.4(0-14.18)$ & $1(0-1)$ \\
High & $4(2-5)$ & $248.60(3-50,859.50)$ & $1(0-254)$ & $0.99(0-1)$ \\
\hline${ }^{\mathrm{a}} p<0.0001,{ }^{\mathrm{b}} p=0.004$ & & & \\
${ }^{\mathrm{c}} p=0.139,{ }^{\mathrm{d}} p=0.232$ & & & \\
$D S$ modified DISCERN tool, min minimum, max maximum &
\end{tabular}


Table 4 Distribution of the video contents

\begin{tabular}{ll}
\hline Video contents $^{*}$ & Total, $n(\%)$ \\
\hline Symptoms of COVID-19 & $11(23.9)$ \\
Relationship between rheumatological diseases and risk of COVID-19 & $12(26.1)$ \\
Whether to continue the use of existing rheumatological drugs & $19(41.3)$ \\
COVID-19 prevention practices for those with rheumatological disease & $14(30.4)$ \\
The place of hydroxychloroquine in the treatment of COVID-19 & $21(45.6)$ \\
Conditions that should be consulted to a doctor or hospital & $3(6.5)$ \\
Psychological effects of COVID-19 on rheumatology patients & $6(13)$ \\
\hline
\end{tabular}

* More than one topic can be mentioned in a video

$n$ number, $\%$ percentage
The main sources of the high-quality videos were academics/universities, followed by physicians. Low-quality videos were predominantly sourced by patients/independent users and news agencies. In a study of the Zika virus pandemic, Bora et al. [20] reported that the main sources of informative videos were universities and misleading videos were produced by independent users. In contrast to the current study results, news agencies were found to be a source of informative videos. Khatri et al. [19] declared news agencies as the main source of useful videos and individual users as the source of misleading videos. Sahin et al. [23] stated that videos produced by independent users were of lower quality than videos from healthcare professionals. This study reveals the importance of source when reviewing videos to obtain medical information from YouTube. Although there is inconsistency in respect of news agencies, academics/universities and healthcare professionals should be considered the source of high-quality information. In the current study, all the videos from academics/universities were of high quality, but the number of videos was very low. Therefore, universities, academics, and healthcare professionals should be supported and motivated to produce videos that provide accurate, useful, and unbiased medical information.

In addition to video quality, one of the major concerns is that videos providing misleading information may have a greater number of views. Although the results of the current study do not support this concern, low-quality YouTube videos during the Zika virus and H1N1 influenza pandemics were found to attract more Internet users than high-quality videos [20, 21]. YouTube studies conducted on Sjögren's syndrome and rheumatoid arthritis have demonstrated that Internet users tended to view useful videos [15, 24]. Users diagnosed with chronic rheumatic disease such as rheumatoid arthritis and Sjögren's syndrome may be better able to distinguish misleading information compared with general users during an acute pandemic, as they already have a basic knowledge of rheumatological diseases. Additionally, high reputation of academics, universities, and physicians may have led the patients to keep the videos presented by these sources in the foreground. Therefore, high-quality videos may be more viewed.

The most frequently discussed topics in videos were the place of hydroxychloroquine in the treatment of COVID-19 and whether to continue the use of existing rheumatological drugs. Interest in hydroxychloroquine has increased following studies presenting potential positive effects in the treatment of COVID-19 [25, 26]. Another reason for the high frequency is that this attractive topic was presented in almost all of the videos provided by news agencies. The first question in the mind of rheumatology patients after the outbreak of COVID19 has been the condition of current drug treatments. Considering the potential effects of rheumatology drugs on the immune system, it can be understood why this issue has been frequently discussed in the videos.

YouTube studies have some limitations. Although the videos were not evaluated by a single researcher, the scoring is subjective. The videos in a single snapshot were listed, and the evaluations were made of this video list. YouTube is a dynamic platform, and over time new videos are added, commented on, and viewed. The video search was performed only in English. As a video search can be influenced by the search history, this issue was minimized by deleting the browser search history. Geographic location and language potentially affect the search results. Finally, the relatively small sample size can be considered another limitation.

\section{Conclusion}

Although the ratio of high-quality videos was high, a considerable number of low-quality videos were detected. At this point, the importance of video sources becomes evident in order to select high-quality videos and access correct information. Academics, universities, and healthcare professionals should create videos on YouTube to ensure that Internet users can access more useful, high-quality, and accurate information. 


\section{Compliance with ethical standards}

Videos which are publicly available on YouTube were assessed, and no human participants/animals were included. Therefore, Ethics Committee approval was not required for this study.

Conflict of interest The authors declare that they have no conflict of interest.

\section{References}

1. World Health Organization (2020) Novel Coronavirus-China. http://www.who.int/csr/don/12-january-2020-novel-coronaviruschina/en/. Accessed 7 February 2020

2. World Health Organization (2020) Novel coronavirus (2019$\mathrm{nCoV}$ ) situation report 17th. https://www.who.int/docs/defaultsource/coronaviruse/situation-reports/20200206-sitrep-17 ncov. pdf?sfvrsn $=17$ fodca 4 . Accessed 7 February 2020

3. Venerito V, Lopalco G, Iannone F (2020) COVID-19, rheumatic diseases and immunosuppressive drugs: an appeal for medication adherence. Rheumatol Int 40:827-828. https://doi.org/10.1007/ s00296-020-04566-9

4. Favalli EG, Biggioggero M, Meroni PL (2014) Methotrexate for the treatment of rheumatoid arthritis in the biologic era: still an "anchor" drug? Autoimmun Rev 13:1102-1108. https://doi.org/10. 1016/j.autrev.2014.08.026

5. Kwok TM, Singla AA, Phang K, Lau AY (2017) YouTube as a source of patient information for varicose vein treatment options. J Vasc Surg Venous Lymphat Disord 5:238-243. https://doi.org/10. 1016/j.jvsv.2016.10.078

6. Tolu S, Yurdakul OV, Basaran B, Rezvani A (2018) Englishlanguage videos on YouTube as a source of information on selfadminister subcutaneous anti-tumour necrosis factor agent injections. Rheumatol Int 38:1285-1192. https://doi.org/10.1007/ s00296-018-4047-8

7. Basnet B, Bhattarai S, Khanal A, Upadhyay M, Baruwal A (2019) Quality of YouTube patient information on prostate cancer screening. Proc (Bayl Univ Med Cent) 32:361-363. https://doi.org/10. 1080/08998280.2019.1594493

8. Ward M, Ward B, Warren C, Silverstein S, Ray C, Paskhover B, Kornitzer J (2019) The quality of YouTube videos as an educational resource for attention-deficit / hyperactivity. Pediatr Neurol. https://doi.org/10.1016/j.pediatrneurol.2019.04.001 [Epub ahead of print]

9. Kocyigit BF, Akaltun MS (2019) Does YouTube provide high quality information? Assessment of secukinumab videos. Rheum Int 39:1263-1268. https://doi.org/10.1007/s00296-019-04322-8

10. Nagpal SJS, Karimianpour A, Mukhija D, Diwakar M, Andrei B (2015) YouTube videos as a source of medical information during the Ebola hemorrhagic fever epidemic. SpringerPlus 4:457. https:// doi.org/10.1186/s40064-015-1251-9

11. Sampson M, Cumber J, Li C, Pound CM, Fuller A, Harrison D (2013) A systematic review of methods for studying consumer health YouTube videos, with implications for systematic reviews. PeerJ 1:e147. https://doi.org/10.7717/peerj.147

12. iProspect Search Engine User Behaviour Study. district4.extension.ifas.ufl.edu/Tech/TechPubs/
WhitePaper_2006_SearchEngineUserBehavior.pdf. Accessed 26 January 2012

13. Rittberg R, Dissanayake T, Katz SJ (2016) A qualitative analysis of methotrexate self-injection education videos on YouTube. Clin Rheumatol 35:1329-1233. https://doi.org/10.1007/s10067-0152910-5

14. Pons-Fuster E, Ruiz Roca J, Tvarijonaviciute A, López-Jornet P (2020) YouTube information about diabetes and oral healthcare. Odontology 108:84-90. https://doi.org/10.1007/s10266-01900445-3

15. Singh AG, Singh S, Singh PP (2012) YouTube for information on rheumatoid arthritis-a wakeup call? J Rheumatol 39:899-903

16. Bernard A, Langille M, Hughes S, Rose C, Leddin D, Veldhuyzen van Zanten S (2007) A systematic review of patient inflammatory bowel disease information resources on the world wide web. Am J Gastroenterol 102:2070-2077

17. Nason GJ, Kelly P, Kelly ME, Burke MJ, Aslam A, Giri SK, Flood HD (2015) YouTube as an educational tool regarding male urethral catheterization. Scand J Urol 49:189-192. https://doi.org/10.3109/ 21681805.2014.975837

18. Esen E, Aslan M, Sonbahar BÇ, Kerimoğlu RS (2019) YouTube English videos as a source of information on breast self-examination. Breast Cancer Res Treat 173:629-635. https://doi.org/10. 1007/s10549-018-5044-z

19. Khatri P, Singh SR, Belani NK, Yeong YL, Lohan R, Lim YW, Teo WZ (2020) YouTube as source of information on 2019 novel coronavirus outbreak: a cross sectional study of English and mandarin content. Travel Med Infect Dis 20:101636. https://doi.org/10. 1016/j.tmaid.2020.101636 [Epub ahead of print]

20. Bora K, Das D, Barman B, Borah P (2018) Are internet videos useful sources of information during global public health emergencies? A case study of YouTube videos during the 2015-16 Zika virus pandemic. Pathog Glob Health 112:320-328. https://doi.org/ 10.1080/20477724.2018.1507784

21. Pandey PN, Singh M, Sood A, Singh G (2010) YouTube as a source of information on the H1N1 influenza pandemic. Am J Prev Med 38:e1-e3. https://doi.org/10.1016/j.amepre.2009.11.007

22. Villafañe JH, Cantero-Tellez R, Valdes K, Usuelli FG, Berjano P (2018) Educational quality of YouTube videos in thumb exercises for carpometacarpal osteoarthritis: a search on current practice. Hand (NY) 13:715-719. https://doi.org/10.1177/ 1558944717726139

23. Şahin A, Şahin M, Türkcü FM (2019) YouTube as a source of information in retinopathy of prematurity. Ir J Med Sci 188:613617. https://doi.org/10.1007/s11845-018-1902-2

24. Delli K, Livas C, Vissink A, Spijkervet FK (2016) Is YouTube useful as a source of information for Sjögren's syndrome? Oral Dis 22:196-201. https://doi.org/10.1111/odi.12404

25. Colson P, Rolain JM, Lagier JC, Brouqui P, Raoult D (2020) Chloroquine and hydroxychloroquine as available weapons to fight COVID-19. Int J Antimicrob Agents:105932. https://doi.org/10. 1016/j.ijantimicag.2020.105932 [Epub ahead of print]

26. Misra DP, Agarwal V, Gasparyan AY, Zimba O (2020) Rheumatologists' perspective on coronavirus disease 19 (COVID19) and potential therapeutic targets. Clin Rheumatol. https://doi. org/10.1007/s10067-020-05073-9 [Epub ahead of print]

Publisher's note Springer Nature remains neutral with regard to jurisdictional claims in published maps and institutional affiliations. 